\title{
Pragmatical solving of uncertainties on production flow
}

\author{
Daniela Gîfu ${ }^{1,2}$, Mirela Teodorescu \\ '„Alexandru Ioan Cuza” University, Faculty of Computer Science, \\ 16, General Berthelot St., 700483, Iaşi \\ daniela.gifu@info.uaic.ro \\ ${ }^{2}$ University of Craiova, Center for Advanced Research in Applied Informatics, 13, A.I. Cuza St., 200585, Craiova, Romania
}

\begin{abstract}
Achieving a high quality product corresponding consumer requirements involves organizing and supporting the production process with a suitable and flexible logistics process. As the process / production flow is better organized, divided into subdomains (production, maintenance, quality), each with clearly defined indicators and responsibilities, the more the process will be more stable, easier to follow and to intervene on perturbations that can appear. In a stable system disturbance variables can be controlled, thus losses can be reduced. Everybody is looking for "the optimal process", "best method" that would remove the uncertainty, ambiguity, defect, doubt, error.

This study aims to present ways of correcting the uncertainties arising in production systems through standardization, communication, waste awareness, efficient logistics, states incertitude interpretation, analysis and performance indicators, applying neutrosophy theory, because a product that has several options increases the grade of uncertainty in the manufacturing process.
\end{abstract}

Keywords: production flow; uncertainty; neutrosophy; logistic; communication procedure;

\section{INTRODUCTION}

Market is designed to provide products to consumers, the same product is in several variants, the consumer has the possibility to choose the desired product according to the qualityprice ratio. Of course a quality product is backed by a steady process, a controlled process by the error in preset limits, advanced equipment that maintain stability, synergy between the production process, equipment maintenance, product quality assurance, logistics processes, human resources. As more complex product is, also manufacturing procedures, quality control, equipment maintenance, personal training more complex are.

The automotive industry is one of the most complex because one type of product can be manufactured in many variants, body color, color harmony with seats and interior, engine type, gearbox type, tire type, mirrors type, roof ... choices and options. Each option generates an uncertainty on the production line, thus having 10 options can produce $2 * * 10$ i.e. 1024 versions of the same model in one shift of day. There are many plants that confront this production 
volume. This situation requests an optimal production flow logistic. In principle all plants use the same concept for over 100 years, Henry Ford invented and it was improved along time. To see the system disorder it is important to have the same type of indicators, production process standardization, either maintenance, or quality, or human resources, or logistics standardization. There are indicators that relate to security and safety work, product quality, production volume, product costs, maintenance, human resource, environment and others. These indicators are followed as part of the process of each department integrated as a system. The system is divided into input, output, processing and feedback. By parameters setting, can be determined the target deviations and threats that lead to uncertainties, defects, deviations, errors.

\section{LOGISTICS}

Logistics is the field of study focused on the design, control, and implementation of the efficient flow and storage of goods and services, and other related information from the point of origin to the point of final consumption with an aim to satisfy the requirements of its existing and prospective customers.

The management of logistics involves the integration of information, transportation, inventory, warehousing, material handling, packaging, and often security.

\section{Physical Product Flow}

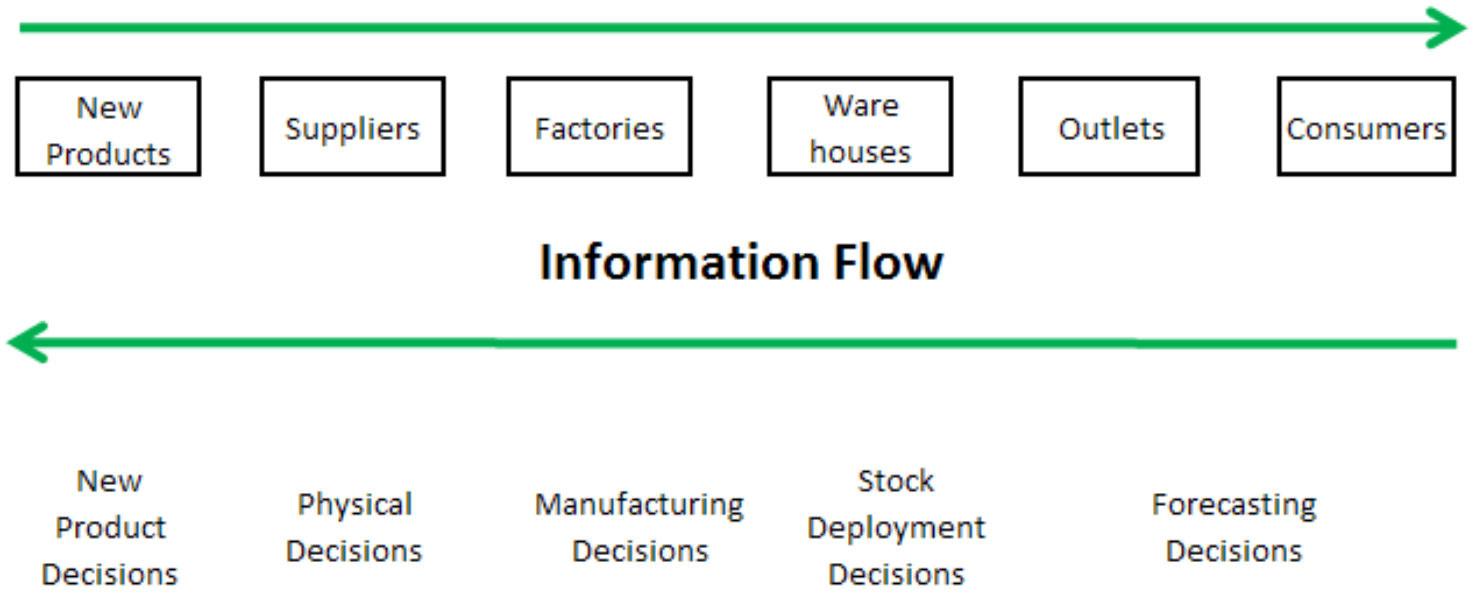

Fig. 1

Logistics management represents the supply chain management component that is used to meet customer demands through the planning, control and implementation of the effective movement and storage of related information, goods and services from origin to destination. Logistics management supports companies to reduce expenses and enhance customer service. Logistics management: understands the role of logistics in an enterprise; defines customer 
service; describes electronic and other logistics information systems; explains inventory management; explains materials management with special reference to Japanesse systems; defines supply chain management; explains methods of transport; illustrates the calculation of transport cost; illustrates the types and cost of warehousing; explains electronic aids in materials handling cost and price determination of purchases; explains how logistics can be organised; explains methods for improving logistics performance;

Information System is an applied discipline that studies the processes of the creation, operation, social contexts and consequences of systems that administrate information. Creation and operation of such systems requires the sub-processes of systems analysis, design, development and management which are bracketed at the beginning.

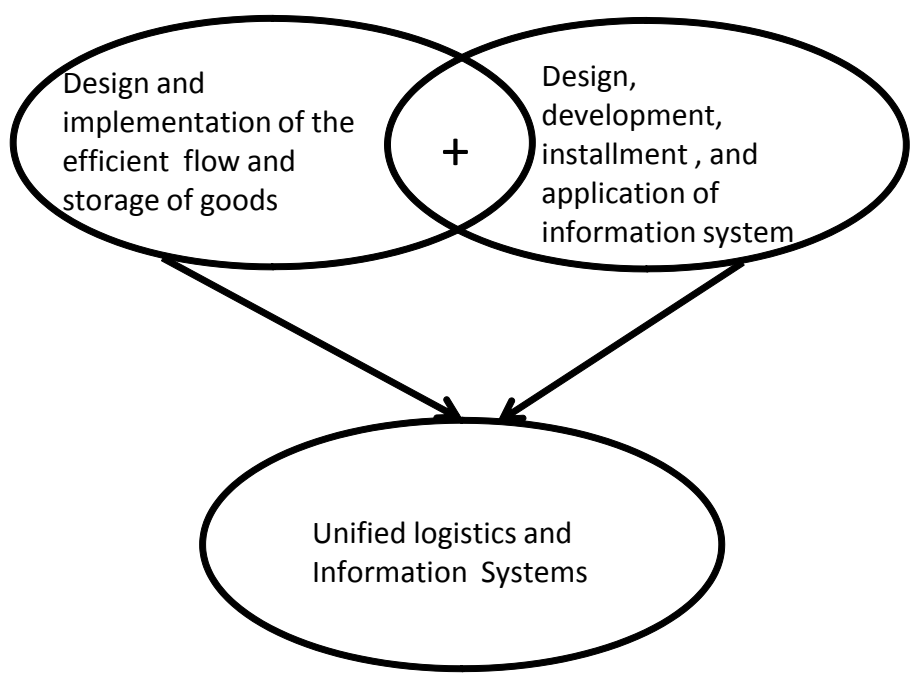

\section{Logistics Information Systems}

Fig. 2

Logistics is the field of study that focuses on the design and implementation of the efficient flow and storage of goods from the point of origin to consumption. Information System is the field of study to deal with problems against the design, development, implementation, application of information system. Logistic Information System (LIS) is the discipline that unifies Logistics and Information Systems.

Information acts in all logistic functions, holding the systems together and coordonating all components of logistics operations, having two major components: planning and coordination; operation. 


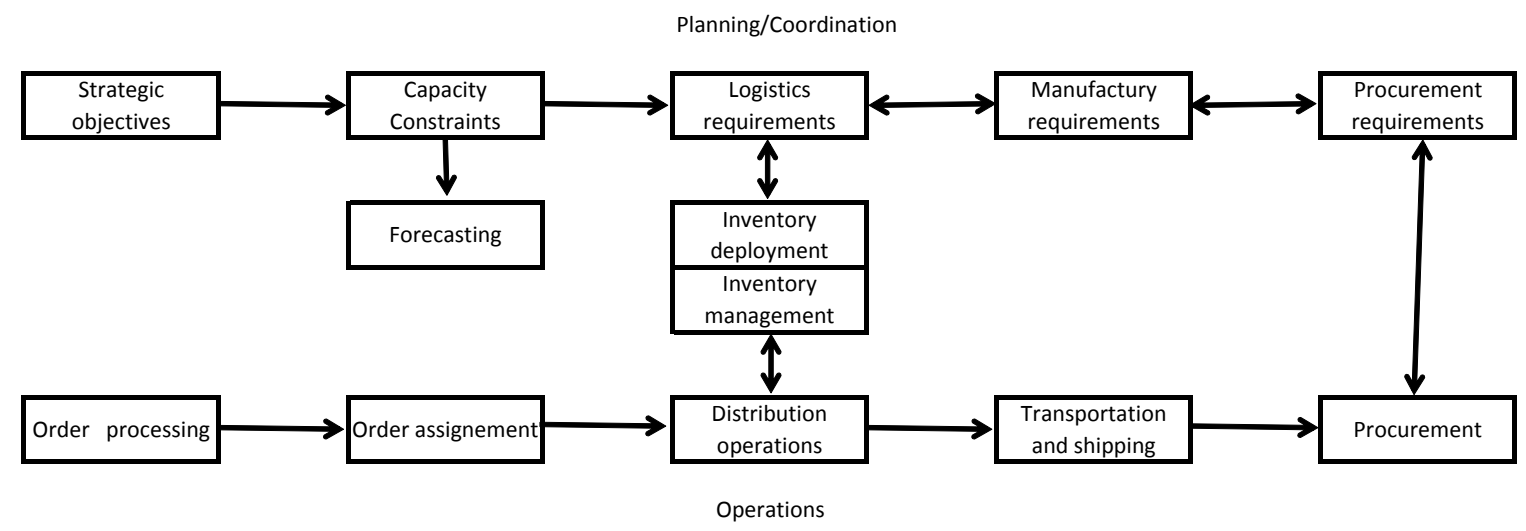

Logistic information requirements

Fig.3

The primary drivers of supply-chain operations are strategic objectives from marketing and financial goals. These initiatives detail the nature and location of customers that supply chain to support inventory, receivables, facilities, equipment, and capacity operations seek to match planned products and services. This will include customer bases, breadth of products, services and promotions. The financial aspect of strategic plan details resources that are required (Farahani, Rezapour, Kardar, 2011).

Capacity constraints identify manufacturing and market distribution limitation, barriers, or bottlenecks. It also helps to identify when specific manufacturing or distribution work should be outsourced. The output of capacity constraint planning is time-phased objectives that detail and schedule facility utilization, financial resources, and human requirements. For each product capacity plans determine the where, when and how much for production, storage, and movement. Using inputs form forcasting, promotional scheduling, customer orders, and inventory status, logistic requirements identify the specific work facilities, equipment, and labour forces required to support the strategic plan. Logistic requirements must be integrated with both capacity constraints and manufacturing requirements to achieve the best performance.

Inventory deployment interfaces with inventory management between planning and coordinating and operations as shown in Fig.3, (Farahani, Rezapour, Kardar, 2011) .

The deployment plan details the timing of where inventory will be positioned to efficiently move inventory through the supply chain. From the information perspective, deployment specifies what, where, and when actions are appropriate for the logistics processes. Inventory management is performed from a day to day basis. Manufacturing requirements determine planned schedules. The traditional deliverable is a statement of time phased inventory requirement that is used to drive master production scheduling (MPS) and manufacturing required planning (MRP).

The involvement of logistics leads to satisfying the client and reducing costs. In order to accomplish this performance, it is recommended to use four organizing principles, namely: 
knowing and accelerating the flows of information; knowing and accelerating the flows of products; knowing and complying with the mutual commitments; knowing and managing the risks (Dima, Vladutescu, 2012).

The points of view regarding the evaluation of logistics around the assessment are: the components which logistics acts upon; the suggestion of a modality of action; the four logistical subsystems; the three possible levels of action; the size of the logistical family in the company; the logistical organization in the company.

The three bases of logistics and their origins

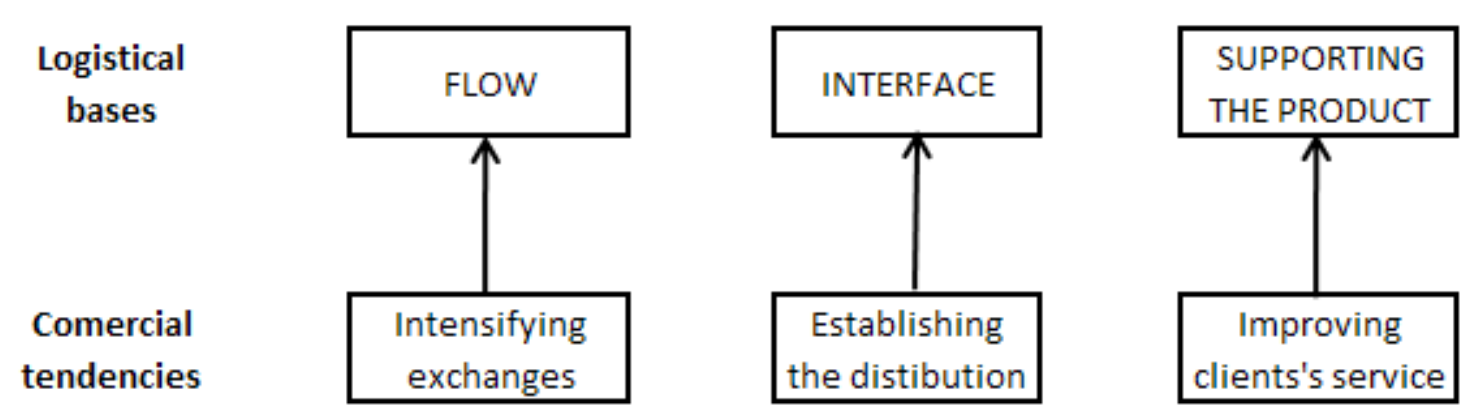

Fig. 4

Taking into account the commercial evolutions, the three components (dimensions) of the operational management can be highlighted, which logistics is based upon, such as: management of the flows necessary for answering to the intensification of the commercial exchanges; the management of the interfaces used for responding to the problems occurred and creation of networks; the management of for sustaining the product, having as aim the improvement of the service at the client (Fig. 4), (Dima, Vladutescu, 2012).

\section{LEAN MANUFACTURING}

In order to gather the production system tools/elements and processes to support a lean manufacturing environment we have to take into consideration three kind of aspects: physical, operational and cultural. Logistics has an important role for: safety, sign-in sheet, objectives/observations, evaluation, housekeeping, agenda, parking lot, ground rules (Dima, Vladutescu, 2012).

Ground rules suppose start and stop on time, be open minded and honest, one conversation at a time, take responsibility for yourself, no rank in class, everyone is entitled to their own opinion, challenge your own personal thinking, there are no dumb questions. Objectives/outcomes suppose that the participants to be able to have a general understanding of the Value Stream Mapping Process, to identify the 7 wastes, understand what policy deploy is 
and how it affects everyone, to have a general understanding of what is a Master Schedule (Tenescu, Teodorescu, 2014).

For many, lean is the set of "tools" that assist in the identification and steady elimination of waste (Teodorescu, Gifu, Ionescu, 2014). If the wastes are eliminated, the quality improve production time and costs are reduced. To accomplish this goal are implemented many procedures, methods, rules such as: Value Stream Mapping (method for analyzing the current state and designing a future state for the series of events that take a product or service from its beginning through the customer), 5S (a list of operations: Sort, Systematize, Shine, Standardize, Self-discipline), Kanban (scheduling system for lean and just-in-time (JIT) production), pokayoke (error - proofing, active involvement by workers in trouble shooting and problem solving to improve quality and eliminate waste), Total Productive Maintenance (a concept to increase the productivity of plant and equipment), one piece flow, small batch production, synchronized to shipping schedules, defect prevention rather than inspection and rework, production planning driven by customer demand, team based work organizations with multi skilled operators empowered to make decisions and improve operations, close integration of the whole value stream: supplier\&customer (Womack James P, Daniel T. Jones, 2003; Tenescu\&Teodorescu, 2014 ).

Lean culture. A traditional organization is characterized by top down management, weak feedback, poor ownership and initiative, limited improvement activity, diluted communication, narrow roles and responsibilities, poor utilization of resources while a lean organization, affects whole organization, non value supports value add, two way communication, roles and responsibilities are clear and broad, improvement driven by all levels, work group focuses on internal improvements (Dima, Vladutescu, 2012; Tenescu, Teodorescu, 2014).

Lean is a culture of continuous improvement practiced at every level of the organization and by every team, lean means respect for people, lean is the elimination of waste in all its forms, lean is add value to your customers, lean is a work environment that assures the quality and safety of all work for both clients and staff, lean is a focus on improving the work process and not on blaming people or creating fear, lean is a culture of teamwork, sharing responsibility, lean is a culture that returns the joy to work, lean is flow, from beginning to end without interruption.

\section{Fundamental concepts of lean manufacturing}

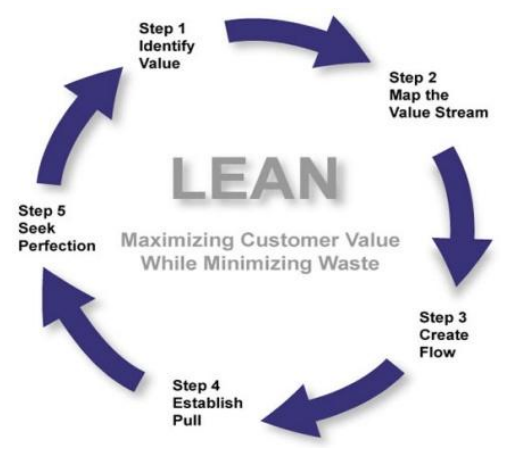

Fig. 5 
Step 1. Identify Value: in Lean Production, the value of a product is defined solely by end user customer, the product must meet the customer's needs at both a specific time and price, to view value through the eyes of the customer requires most companies to undergo difficult and comprehensive reorganization of people, their mindset and behaviors and business processes, specifying value in interpersonal relationship means simply to understand the wants and expectations of the people that we interact with (Dima,Vladutescu, 2012; Teodorescu, Gifu, Ionescu, 2014; Tenescu, Teodorescu, 2014).

Step 2. Map the Value Stream: identifying the value in lean production means to understand all the activities required to produce a specific product, and then to optimize the whole process from the view of the end-user customer, identifying the value stream in individual or group behavior means to understand what people do and why they do it (Tenescu, Teodorescu, 2014).

Step 3. Create Flow: In lean production means to process parts continuously, from raw materials to finished goods, one operation or one piece at a time; In behavioral context means to behave in a manner that minimizes or eliminates delays or stoppages in the work performed by others.

Step 4. Establish Pull: the concept of pull in lean production means to respect the pull, or demand, of the customer; in behavioral context means to recognize that people operate under many different mental models which requires us to adjust our style or approach often; forecasting the behaviors of others is pure waste because it is time consuming and often inaccurate, and should thus be eliminated. Practicing lean behaviors reduces ambiguity and re-work in interpersonal relationships (Tenescu, Teodorescu, 2014).

Step 5. Seek Perfection: in lean production means that there are endless opportunities for improving the utilization of all types of assets; in behavioral context means to take advantage of the transparency brought about by the first four steps in order to more easily identify and eliminate behaviors that do not create value (Bob Emiliani,2008).

Lean implementation is therefore focused on getting the right things to the right place at the right time in the right quantity to achieve perfect work flow, while minimizing waste and being flexible and able to change. The flexibility and ability to change are within bounds and not openended, and therefore often not expensive capability requirements. More importantly, all of these concepts have to be understood, appreciated, and embraced by the actual employees who build the products and therefore own the processes that deliver the value (Mark Rosenthal , 2002).The cultural and managerial aspects of lean are possibly more important than the actual tools or methodologies of production itself. There are many examples of lean tool implementation without sustained benefit, and these are often blamed on weak understanding of lean throughout the whole organization.

Value added $=$ processes that change the product fit, form, function to meet customer specifications; work that customer is willing to pay for (Teodorescu, Gifu, Ionescu, 2014). 
Waste $=$ the element of production that adds no value to the product, adding only cost and/or time; work that the customer is not willing to pay for.

The original seven wastes are:

- Transport (moving products that are not actually required to perform the processing)

- Inventory (all components, work in process and finished product not being processed)

- Motion (people or equipment moving or walking more than is required to perform the processing)

- Waiting (waiting for the next production step, interruptions of production during shift change)

- Overproduction (production ahead of demand)

- Over processing (resulting from poor tool or product design creating activity)

- Defects (the effort involved in inspecting for and fixing defects),(Womack, Jones, 2003; Tenescu, Teodorescu, 2014)

\section{MAINTENANCE INDICATORS}

In automatical systems equipments operates in cycles time defined as sum of status: Cycling time, Starved time, Blocked time, Waiting aux part time, Waiting attention time, Repair in progress time, Emergency stop time, Bypass time, Tool change time, Setup time, Break time, No comunications, Fig. 6. These statuses are defined in PLC for process analyse and evaluation. Related on these statuses are proceeded also the maintenance indicators.

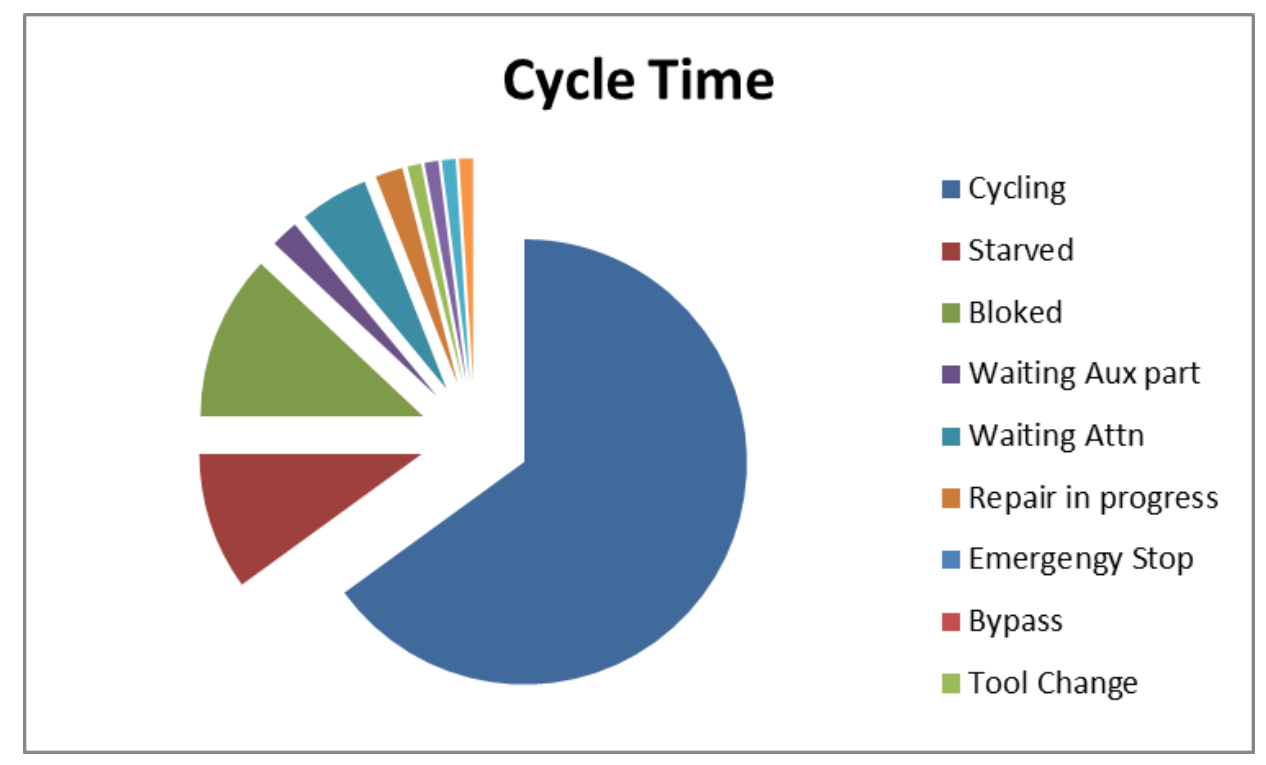

Fig. 6

The OEE is measured as : (Availability)*(Performance)*(Quality) Where:

- Availability: The portion of the OEE Metric that represents the percentage of scheduled time that the operation is available to operate. Often is referred as Uptime. 
- Performance: The portion of the OEE Metric that represents the speed at which the Work Center runs as a percentage of its designed speed.

- Quality: The portion of the OEE Metric that represents the Good Units produced as a percentage of the Total Units Started.

Definition of a failure: A failure is declared when the equipment does not meet its desired objectives. Therefore, we can consider any equipment that cannot meet minimum performance or availability requirements to be "failed". Similarly, a return to normal operations signals the end of downtime or system failure, is considered to be "non-failed".

Mean Time to Repair (MTTR): This is the mean time the facility is in the status of "Repair".

Calculation:

MTTR = Repair in Progress Time $(\mathrm{min}) /$ Repair in Progress Occurrences.

Mean Time Between Failures (MTBF): This shows the amount of time the machine spends in production time as a percentage of all the states except Break and No Communications.

Calculation:

MTBF $=($ Time in Auto $/$ Total Time $) \times 100$

Where

Time in auto $=$ Cycling Time + Blocked Time + Starved Time + Waiting Auxiliary Time + Bypass Time

and

Total Time $=$ Cycling Time + Blocked Time + Starved Time + Waiting Auxiliary Time + Bypass Time + Tool Change Time + Waiting Attention Time + Shutdown Time + Emergency Stop Time + Set Up Time

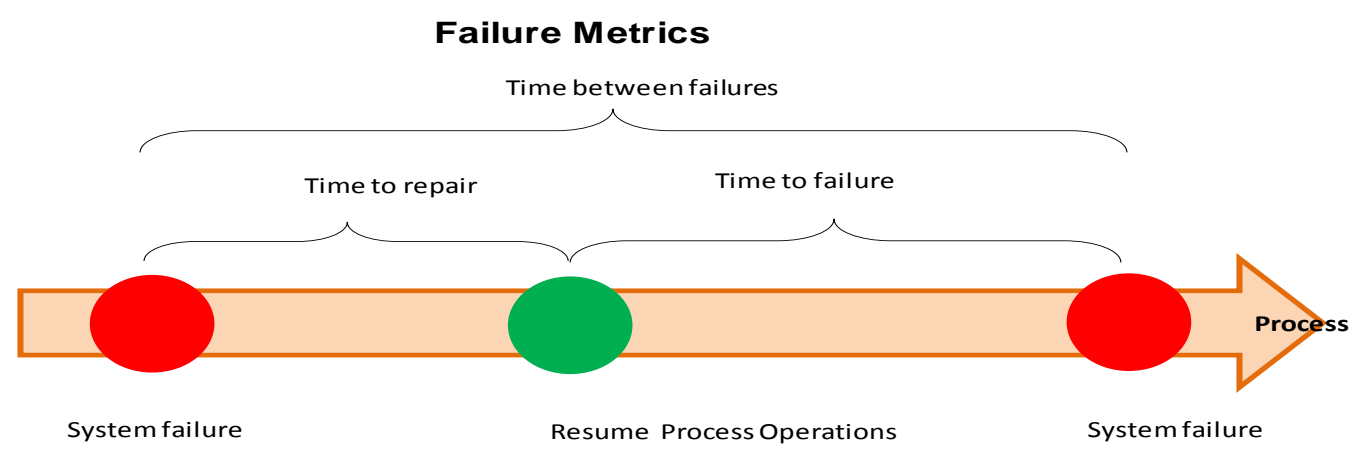

Fig. 7

A process is stable when there is no variability in the system, when the outcome is by design, as expected. The systems variation we are talking about in this study refers to uncertainty, confusion that can occur in various situations in the manufacturing process so that, it will lead to another product than expected one or a scrap. Theory that manage uncertainty, 
neutrality was developed by F. Smarandache, professor of mathematics at the University of New Mexico and is named Neutrosophy.

It has to be mentioned that in time, neytrosophy evolution looks like: Zadeh introduced the degree of membership/truth (t) in 1965; Atanassov introduced the degree of nonmembership /falsehood (f) in 1986; Smarandache introduced the degree of indeterminacy/neutrality (i) as independent component in 1995 (published in 1998).

Neutrosophy as an analytical study, is related to multiple-valued logic because at one moment one shows that a statement $\langle\mathrm{A}\rangle$ was proved true by a philosopher $\mathrm{X}$ whereas latter another philosopher $\mathrm{Y}$ proved the opposite statement $<$ Anti-A $>$ was true. Therefore, both $\langle\mathrm{A}\rangle$ and $\langle$ Anti-A $\rangle$ were true. \{Whence one can deduce that both $\langle A\rangle$ and $\langle$ Anti-A $\rangle$ could be false. Even more, using a neutrosophic interpretation, one could say that other ideas in between $\langle A\rangle$ and $\langle$ Anti-A $\rangle$ and related to them, noted by $\langle$ Neut-A $\rangle$, could be true as well. This relates to dialetheism, which says that some contradictions are true, to paraconsistent logic, to intuitionist logic, till neutrosophic logic (where $\langle A\rangle$, $\langle$ Anti-A $\rangle$, and ideas in between them belonging to $<$ Neut-A $>$ could all be true or partially true) (Smarandache, 2005).

In a process, practically can occur such situations when we are put in a position of uncertainty that leads to the process variation by instability, by errors. Below are presented two methods of analysis, evaluation and correction of the process: the Ishikawa diagrams and Pareto chart.

Ishikawa diagrams (also called fishbone diagrams, cause-and-effect diagrams) are causal diagrams created by Kaoru Ishikawa (1968) that shows the causes of a specific event (Womack, James P, Daniel T. Jones, and Daniel Roos,1990; Holweg, Matthias 2007). Common uses of the Ishikawa diagram are product design and quality defect prevention, to identify potential factors causing an overall effect. Each cause or reason for imperfection is a source of process variation. Causes are usually grouped into major categories to identify these sources of variation such as: people, methods, machines, materials, measurements, environment (Ishikawa, Kaoru, 1976).

Related to these categories can be extended to detailed items like anyone involved with the process, how the process is performed and the specific requirements for doing it, policies, procedures, rules, regulations and laws, any equipment, computers, tools, etc. required to accomplish the job, raw materials, parts, pens, paper, etc. used to produce the final product, data generated from the process that are used to evaluate its quality, the conditions, such as location, time, temperature, and culture in which the process operates (Juran, J. M., \& Gryna, F. M. (1970). 


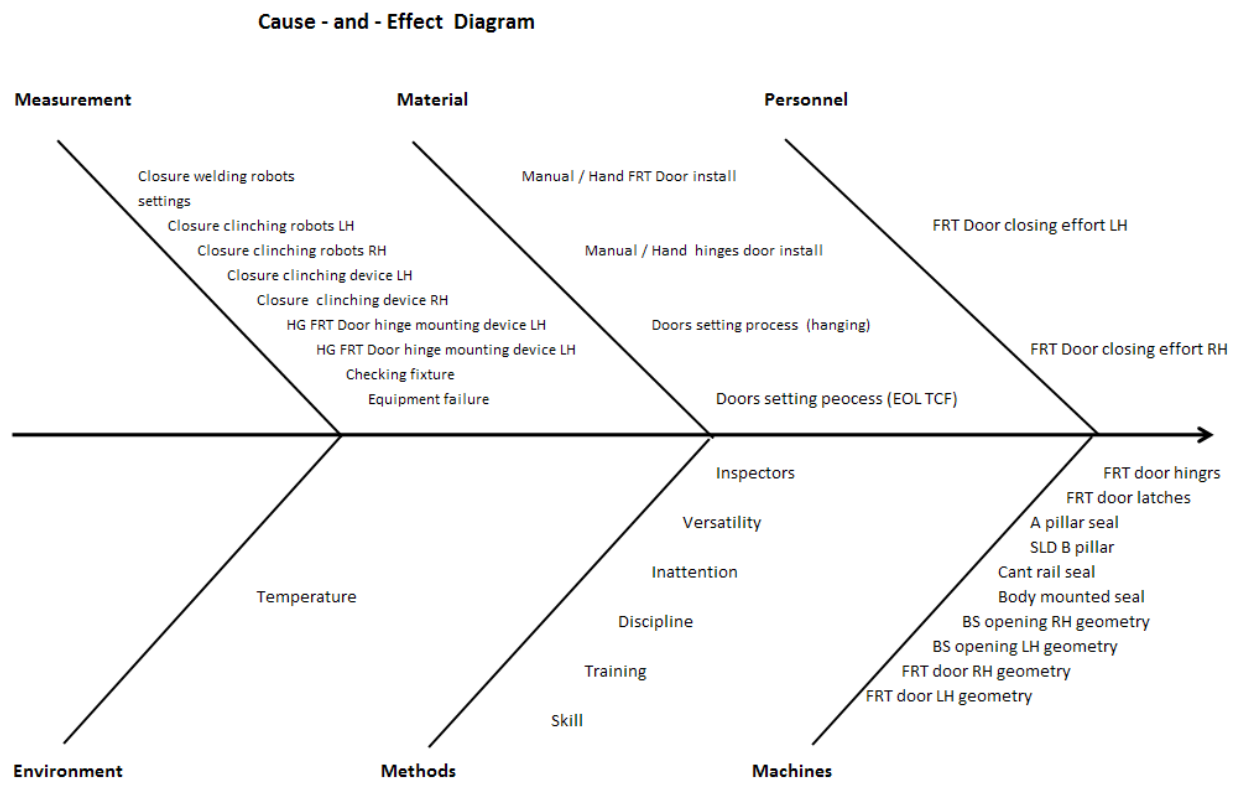

Fig. 8

Pareto analysis is a statistical technique in decision-making used for the selection of a limited number of tasks that produce significant overall effect. It uses the Pareto Principle (also known as the $80 / 20$ rule) the idea that by doing $20 \%$ of the work you can generate $80 \%$ of the benefit of doing the entire job.

\section{How to use the Pareto charts}

Step 1: Identify and list problems - that occur in manufacturing process with the highest frequency and concern the process.

Step 2: Identify the root cause of each problem - for each issue it is important to identify the fundamental cause. The used methods can be: Brainstorming, 5 Whys, Cause and effect analysis, and Root cause analysis.

Step 3: Score problems - scoring each problem depends on the sort of problem that it has to be solved, for quality, safety, efficiency, cost.

Step 4: Group problems together by root cause - similarly problems belong to the same group.

Step 5: Add up the scores for each group - assign scores to each group of problems.

Step 6: Take action - is the moment to deal with the top priority problem, group of problems and also the purpose that you want (Montgomery, 1985). 


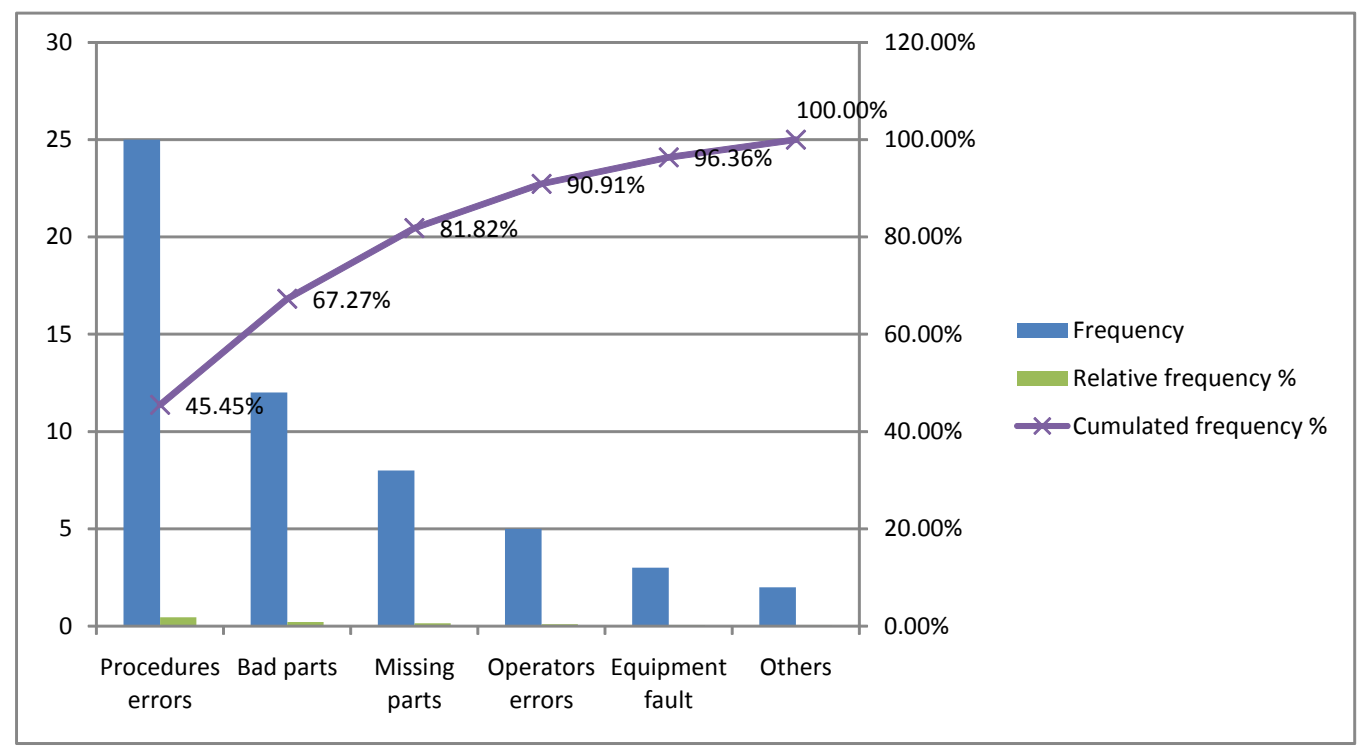

Fig. 9

In this example there are few issues that appear in process. Examining "Operators errors" we can make the decision that human errors can be diminished by an IT application, automatisation, to reduce human decision. It is true that in a process in which can appear confusion of choicing the appropriate part (for example between left and right), it can generate errors. Automatisation of process can avoid human error, sustained by appropriate IT applications, andons, operators training. Analysing a cause that generates $20 \%$ of errors, and eliminate it by investing in process, it can solve $80 \%$ of issues.

The emergence of RFID (Radio Frequency Identification) technology has been greatly increased efficiency in the production process management, material flow management, logistics and transport, retail and distribution, and other fields of the national economy industries, including electronic information industry. RFID may eventually replace the ubiquitous bar code in the future and become the main technology in logistics and supply chain management field (Singer, 2006).

Compared with the popular bar code technology, electronic tag has many advantages: omitting the manual control, waterproofing, antimagnetic, bearing the high temperature, a long service life and wide reading distance. Moreover, on the electronic label, data may encrypt, the storage capacity is big and the canned data can be changed. Thus, it has wider and more convenient application than the bar code. The popularization and the application of RFID will bring revolutionary changes to the retail and logistics industry.

Automotive industry uses RFID advantages in production flow, in automatical process for safety and security of manufacturing management. 


\section{CONCLUSIONS}

According to the reason to have a stable process, accuracy products, efficiency flow of the process it is important to solve the uncertaints, all types and from all places. Applying organizational methods such as "lean manufacturing", standardization of indicators or IT technology are only few samples. Each producer has its own method to implement improving concepts derived from above mentioned. TPS (Toyota Production System) is the basically concept, based on principles such as continuous improvement, respect for the people, long-term philosophy, the right process will produce the right results, add value in organization, continuous solving root problems drives organizational learning.

\section{Reference}

[1] Reza Zanjirani Farahani, Shabnam Rezapour, Laleh Kardar, Logistics Operations and Management: Concepts and Models, 2011 Elsevier Inc.

[2] Ţenescu A, Teodorescu M. Lean Manufacturing: a concept towards a sustainable management, Communications in Applied Sciences, Infinity Press. Vol.2, No.1, 2014, 97-110

[3] Holweg M. The genealogy of lean production. Journal of Operations Management. 2007, 25 (2): 420-437. doi:10.1016/j.jom.2006.04.001

[4] Bailey D. Automotive News calls Toyota world No 1 car maker. Reuters.com. Reuters. Retrieved 19 April 2008.

[5] Vlăduțescu S. Principle of the Irrepressible Emergence of the Message. Jokull, 2013, 63(8), 186-197.

[6] Womack JP, Jones DT, Roos D. The Machine That Changed the World. 1990. Simon \& Schuster Riverside, New Jersey, USA.

[7] Dima IC, Vlăduţescu S. Persuasion elements used in logistical negotiation: Persuasive logistical negotiation. 2012. Saarbrucken: LAP Lambert Academic Publishing.

[8] Krafcik JF Triumph of the lean production system. 1988. Sloan Management Review.

[9] Emiliani ML, Lean Behaviors. 1998. LLC, Wethersfield, CT, USA.

[10] Ohno T, Toyota Production System. 1988. Productivity Press.

[11] Vlăduţescu Ş, Reengineering some uncertainties about communicational relationships. International Letters of Social and Humanistic Sciences. 2014 (14) 73-81.

[12] Going CB, Preface to Arnold and Faurote. Ford Methods and the Ford Shops. 1915.

[13] Ford H, Crowther S. My Life and Work. 1922. Garden City, New York, USA: Garden City Publishing Company, Inc.

[14] Womack JP, Jones DT. Lean Thinking. 2003. Free Press.

[15] Vlăduţescu Ş, Ciupercă EM. Next Flood Level of Communication: Social Networks. 2013. Aachen: Shaker Verlag.

[17] Grabara J, Kolcun M, Kot S. The role of information systems in transport logistics. International Journal of Education and Research. 2014. 2(2). 
[18] Bicheno J, Holweg M. The Lean Toolbox. 2009. PICSIE.

[19] Hopp W, Spearman M. Factory Physics: Foundations of Manufacturing Management. 2008. Waveland Press, Inc.

[20] Vlăduţescu Ş. Eight Computational-Communicative Operations of Building Information. Mitteilungen Klosterneuburg. 2014. 64(1).

[22] Bajdor P, Grabara I. The Role of Information System Flows in Fulfilling Customers' Individual Orders. Journal of Studies in Social Sciences. 2014. 7(2).

[23] Vlăduţescu Ş. Three Diachronic Paradigms of Communication. International Journal of Education and Research, 2013. 1(12).

[26] Dima IC, Vlăduţescu Ş. Risk Elements in Communicating the Managerial Decisions. European Journal of Business and Social Sciences. 2012. 6(1), 27-33.

[28] Smarandache F, Vlăduţescu Ş. Communication vs. Information, a Neutrosophic Solution. Neutrosophic Sets and Systems. 2013. 1.

[29] Smarandache F, Vlăduţescu Ş. Neutrosophic Emergences and Incidences in Communication and Information. 2014. Saarbrucken: LAP Lambert Academic Publishing.

[31] Colhon M. Automatic Lexical Alignment between Syntactically Weak Related Languages. Application for English and Romanian. 2013. Computational Collective Intelligence. Technologies and Applications (pp. 266-275). Springer Berlin Heidelberg.

[45] Womack J. Gemba Walks. 2011. Lean Enterprise Institute, Inc.

[46] Vlăduţescu Ş. Silence as an Uncertainty Communicational Inductor. International Letters of Social and Humanistic Sciences. 2014. (14).

[48] Grabara J, Modrak V, Dima IC. Sustainable Logistics and Business 2014.

[53] Rosenthal MS. ME Lean Directions Newsletter, 2002.

[54] Teodorescu M, Nicolescu A. International Letters of Social and Humanistic Sciences 2(1) 2015 48-59.

[55] Teodorescu M, Marcincin JM, Gîfu D. International Letters of Social and Humanistic Sciences 27 (2014) 22-33

[56] Teodorescu M, Gîfu D, Ionescu D. International Letters of Social and Humanistic Sciences 17 (2014) 61-69.

[57] Teodorescu M, Bosun P, Gifu D. International Journal of Education and Research, Vol.2, No.3, 2014.

[58] Buşu OV, Teodorescu M, Gîfu D, International Letters of Social and Humanistic Sciences 27 (2014) 82-93

[59] Ishikawa Kaoru. Guide to Quality Control. 1976. Asian Productivity Organization.

[58] Juran JM, Gryna FM. Quality planning and analysis. 1970. New York: McGraw-Hill.

[60] Montgomery DC. Statistical quality control. 1985. New York: Wiley.

[61] Montgomery DC. Design and analysis of experiments, 1991. 3rd ed. New York: Wiley.

[62] Gîfu D, Teodorescu M. International Letters of Social and Humanistic Sciences 17(2) (2014) 119-127 
[62] Marcincin JM, Gîfu D, Teodorescu M. International Letters of Social and Humanistic Sciences 29 (2014) 29-34

[64] Holweg M. "The genealogy of lean production." Journal of Operations Management. 2007. 25(2): 420-437.

[65] Singer T, RFID and Logistics: 4 Trends you need to know. 2006. Industrial Maintenance and Plant. 67 (9): 18-19. 